\begin{tabular}{l}
\hline Alimentary Pharmacology \\
\hline \& Therapeutics \\
\hline
\end{tabular}

\title{
Proton pump inhibitor use is associated with an increased risk for microscopic colitis: a case-control study
}

\begin{tabular}{|r|l|}
\hline Journal: & Alimentary Pharmacology \& Therapeutics \\
\hline Wanuscript ID: & APT-0585-2010.R2 \\
\hline Date - Manuscript type: & Original Scientific Paper \\
\hline Author: & $19-$ Aug-2010 \\
\hline & $\begin{array}{l}\text { Complete List of Authors: } \\
\text { Keszthelyi, Daniel; Maastricht University Medical Center, } \\
\text { Gastronterology and Hepatology } \\
\text { Sastronterology and Hepatolongy Gerben; Maastricht University Medical Center, } \\
\text { Gastroenterology and Hepatology } \\
\text { de Kort, Sander; Maastricht University Medical Center, } \\
\text { Gastroenterology and Hepatology } \\
\text { Scholtes, Brian; Orbis Medical Concern, Internal Medicine } \\
\text { Engels, Leopold; Orbis Medical Concern, Internal Medicine } \\
\text { Masclee, Ad; Maastricht University Medical Center, } \\
\text { Gastroenterology and Hepatology }\end{array}$ \\
\hline Keywords: & $\begin{array}{l}\text { Clinical pharmacology < Topics, Large intestine < Organ-based, } \\
\text { NSAIDs < Topics, Outcomes research < Topics }\end{array}$ \\
\hline
\end{tabular}

\section{SCHOLARONE ${ }^{m}$ Manuscripts}


1

2

4

5

6

7

8

9

10

Proton pump inhibitor use is associated with an increased risk for microscopic colitis: a casecontrol study

Running title: Proton pump inhibitors and microscopic colitis

D. Keszthelyi ${ }^{1}$, SV. Jansen ${ }^{1}$, GA. Schouten ${ }^{1}$, S. de Kort ${ }^{1}$, B. Scholtes ${ }^{2}$, LG. Engels ${ }^{2}$, AAM. Masclee $^{1}$

${ }^{1}$ Division of Gastroenterology-Hepatology, Department of Internal Medicine, Maastricht University Medical Centre+, Maastricht, The Netherlands

${ }^{2}$ Division of Gastroenterology, Department of Internal Medicine, Orbis Medical Concern, Sittard, The Netherlands

Correspondence:

Daniel Keszthelyi, MD

Division of Gastroenterology-Hepatology

Department of Internal Medicine, Maastricht University Medical Centre+ Maastricht, The Netherlands daniel.keszthelyi@intmed.unimaas.nl +31433881982 


\begin{abstract}
Background: Microscopic colitis (MC) causes chronic watery diarrhea. Recent studies have suggested an etiological role for various medications, including proton pump inhibitors, in the pathogenesis of MC.

Aim: To determine whether an association exists between microscopic colitis and proton pump inhibitor use in patients with documented microscopic colitis versus age and sex-matched controls.

Methods: In this retrospective case-control study. Cases of microscopic colitis from a secondary and tertiary referral medical center diagnosed in the last 5 years were reviewed. Demographic characteristics, clinical, histological and endoscopic records, as well as exposure to PPIs and NSAIDs were assessed. Controls from the population were matched to cases by gender and by age.

Results: During the investigated period, 136 cases were identified in both hospitals. Of these, 95 cases of MC were retrieved for detailed analysis. Exposure to proton pump inhibitors at the time of the histological diagnosis was significantly higher in MC patients than in controls $(38 \%$ vs $13 \%, p<0.001$; adjusted OR of $4.5[95 \% \mathrm{Cl} 2.0-9.5])$.

Conclusion: This observation confirms the presumed association between microscopic colitis and PPI use and supports the possible etiological role of PPI exposure in the development of microscopic colitis.
\end{abstract}

Keywords: microscopic colitis, proton pump inhibitors 
In the past, MC was thought to be a rare disorder. However, it has recently become apparent that MC accounts for up to $13 \%$ of patients investigated for chronic diarrhea, particularly in middle-aged and elderly patients $(2,3)$. Pardi et al. reported an increasing annual incidence rate of 7.1 for collagenous colitis and of 12.6 for lymphocytic colitis per 1000000 in the period of 1998 to 2001 (3). The cause of MC remains unknown. One postulated hypothesis points to increased colonic permeability in MC patients, thereby allowing luminal antigens to enter the lamina propria and elicit an immune and inflammatory reaction. Environmental risk factors, such as drugs, have been suggested as causes or triggers of this process (4). These drugs include non-steroidal anti-inflammatory drugs (NSAIDs), selective serotonin reuptake inhibitors (SSRIs), biphosphonates, beta-blockers, statins and proton pump inhibitors (PPIs) (5).

Recently, several case reports have been published which further highlight the association between PPI use and development of MC (6-9). Initially, lansoprazole has been found to be associated with a high likelihood of inducing MC (10). A recent study also points to similar effects in case of exposure to omeprazole and esomperazole (9). PPIs are one of the most frequently prescribed classes of medications worldwide because they combine a high level of efficacy with low toxicity. In 2006, expenditure on these drugs was 10 billion USD globally (11). In the five years since the introduction of esomeprazole in 2001, prescriptions for PPIs have doubled (11). A recent socio-demographic study from The Netherlands found that $11.8 \%$ of the population had at least one prescription for a PPI in the 
year 2006 (12). It is noteworthy, that the increasing incidence of MC (3) seems to parallel the rising use of PPIs. In this report, we analyze the possible association between MC and PPI use in patients with documented MC versus age and sex-matched controls.

\section{Methods}

Patients

We performed a retrospective, two-center, observational, case-control study. Patients with MC were identified by reviewing the pathology database and medical records at the Maastricht University Medical Center, The Netherlands, a tertiary medical center, and at Orbis Medical Center, Sittard, The Netherlands, a secondary medical center, from November 2005 to November 2009. Diagnosis was based on clinical symptoms, data from histology and endoscopy. Following identification, written informed consent was obtained from the patients to assess medical and pharmacy records. Information on prescribed medication for the period of two years prior to the histological diagnosis was obtained from the pharmacy database. All patients having had at least one prescription for a PPI (esomeprazole, omeprazole, lansoprazole, pantoprazole or rabeprazole) in 180 days prior to the time of histological diagnosis were considered exposed to PPIs. Similarly, we investigated exposure to NSAIDs, a known risk factor for MC, as well as exposure to benzodiazepines (BDZs), diuretics and ACE inhibitors, which have not been associated with MC, as control medication. Use of low-dose aspirin (up to $300 \mathrm{mg} /$ day) was not considered as NSAID exposure. The reason for selecting a time window of 180 days between drug exposure and diagnosis of microscopic colitis was the considerable delay that can occur between initial symptom onset and eventual endoscopic examination with mucosal biopsy sampling. Also, comorbidity was also assessed by reviewing medical records.

\section{Controls}

The control group consisted of 355 individuals randomly selected from the general population in Maastricht, The Netherlands, derived from the municipal residential database. Controls received questionnaires regarding their current medical status and drug exposure. Ninety-five controls were matched randomly to cases by gender and by age (within 1 year). Also, comorbidity was assessed by reviewing medical records. 


\section{Statistical analysis}

Statistical analysis was done by performing $x^{2}$ test and generalized linear model for binomial regression to calculate ORs and 95\% Cls using SPSS, version 17.0 (Chicago, Illinois). Statistical significance was predetermined as $p<0.05$.

\section{Results}

During the investigated period, 136 cases were identified in both hospitals. Of these, 95 cases of MC were retrieved for detailed analysis. No detailed pharmacy records were available for the remaining 41 cases. Mean age of MC patients was $58 \pm 1$ yrs, $66 \%$ were women. We observed a 1:1.07 ratio for lymphocytic vs collagenous colitis. Cases did not differ significantly in BMI from controls $(25 \pm 5$ vs. $26 \pm 4 \mathrm{~kg} / \mathrm{m}^{2}$, mean $\pm \mathrm{SD}$ ). Exposure to both PPIs and NSAIDs at the time of the histological diagnosis was significantly higher in patients than in controls (PPI $38 \%$ vs $13 \%, p<0.001$ and NSAIDs $20 \%$ vs $5 \%, p=0.002$ vs control, respectively). Co-exposure to both PPIs and NSAIDs was also significantly higher in $\mathrm{MC}$ patients compared to controls ( $8 \%$ vs $0 \%, \mathrm{p}=0.007$ ). After adjustment for concomitant exposure to NSAIDs, BDZs, diuretics and ACE inhibitors as covariates, an adjusted OR of $4.5[95 \% \mathrm{Cl}$ 2.0-9.5] was found for PPI exposure (see table 1). There were no differences observed in exposure to PPIs or NSAIDs with regard to the histological subtype (collagenous or lymphocytic). No significant association was found between gender and histological subtype or drug exposure.

Considering the fact that the pharmacy records were not available or obtainable in case of 41 patients, calculations have been done according to the 'worst-case scenario.' This would mean that of the 41 patients, from which no data on medication was obtainable or available, the assumption was made that none were taking PPIs prior to the histological diagnosis ( 0 out of 41 , overall 36 out of 136). As for the control population for these additional 41 patients, we presumed that the consumption of PPIS would be equal to what we observed in the control population (13\%, approximately 5 out of 41 , overall 17 out of 136). Repeating the binary logistic regression analysis with these fictive numbers results in an OR of $2.5[\mathrm{Cl} 1.3-4.7]$ for PPI exposure. 
Regarding the type of PPI patients were exposed to, we found the following distribution: $40 \%$ omeprazole, $23 \%$ esomeprazole, $28 \%$ pantoprazole, $6 \%$ rabeprazole, $3 \%$ patients used lansoprazole (see table 3).

Celiac disease and autoimmune diseases have been reported to be associated with MC (4). In our patient population, celiac disease was diagnosed in $3 \%$ of patients with MC. This rate is comparable to previous reports (3). Rheumatoid arthritis was found in $6 \%$, hypothyroidism in $5 \%$ of MC patients (see table 2). Multivariate logistic regression analyses with comorbidities as covariates resulted in an OR of 5.8 [2.5-13.5] for PPI exposure. This implies that patients with comorbidity have an even higher risk for developing MC upon exposure to PPIs.

\section{Discussion}

Recent studies have emphasized the association between exposure to PPIs and occurrence of MC. Our findings confirm the importance of considering PPI use as a possible etiological factor for MC. However, case-control studies generally do not allow interpretation with respect to a causal relation. It should be noticed that our study has a number of limitations. First, the population in this study is relatively small and pharmacy records were not available or obtainable in about $30 \%$ of the cases in the period reviewed. Nevertheless, calculations according to a 'worst-case scenario' in which none of the remaining $41 \mathrm{MC}$ patients were exposed to PPIs in the studied period still show a significant risk for developing MC. Second, use of over-the-counter medication could not be assessed. Third, the time window between symptom onset and histological diagnosis may vary largely between MC patients, which makes assessment of drug exposure more difficult. For that reason, we assessed drug intake for a longer time window preceding the diagnosis. Fourth, for similar reasons, history of gastrointestinal symptoms could neither be assessed in this time period. Further studies, when possible on larger samples of patients, are needed to confirm our observations.

The pathophysiological mechanisms underlying PPI-related induction of MC are poorly understood. Proton pumps $\left(\mathrm{H}^{+} / \mathrm{K}^{+}\right.$ATPases $)$are present not only on gastric epithelium but also on colonic epithelium where they contribute to whole body potassium homeostasis (13). Inhibition of the colonic 
proton pumps may therefore affect local electrolyte balance and compromise fluid acidification, which can possibly affect immune reactions in the colonic mucosa.

Proteins other than $\mathrm{H}^{+} / \mathrm{K}^{+}$ATPase have also been reported as targets for PPIs. Omeprazole and lansoprazole have been observed to induce smooth muscle relaxation and to inhibit contractile activity (13). This effect on contractile systems could also affect tight junction functionality since tight junction proteins are directly linked to the actinomyosin cytoskeleton. Therefore, conformational changes in the cytoskeleton of epithelial cells may result in alterations in the function of the tight junction, which leads to increased paracellular permeability. As a result, luminal contents can more easily penetrate the lamina propria causing an immune and/or inflammatory reaction. Esomeprazole has been shown to increase paracellular permeability in the upper gastrointestinal tract in vitro (14) and in vivo in humans (15). Increased paracellular permeability has been observed in $M C$ and is considered a key component in the induction of diarrhea (16). Accordingly, expression of the tight junction proteins occludin, claudin-4 and zonuline-1 (ZO-1) was found to be decreased in mucosal biopsy specimens from MC patients $(16,17)$. We speculate that a direct or indirect effect of PPIs on colonic tight junctions and paracellular permeability may explain the association between PPI use and MC observed in this study.

Although not directly linked to the pathogenesis of MC, recent case reports have related long-time PPI exposure to severe hypomagnesemia $(18,19)$. This effect has been described for omeprazole, esomeprazole, pantoprazole and lansoprazole as well (18). It has been proposed that PPIs induce a defect in the active absorption of magnesium in the intestine. This may result from an effect of PPIs on the tight junction proteins or on the TRPM6 and 7 channels (transient receptor potential melastin 6 and 7). The latter are key molecules involved in active magnesium absorption. Either changes in intestinal $\mathrm{pH}$ induced by PPIs may affect channel functions, or subjects who are heterozygous carriers of TRPM6/7 mutations are more susceptible to developing adverse reactions to PPIs (19). Presently, we cannot exclude that effects of PPIs on magnesium absorption also play a role in the pathogenesis of MC. 
In addition, it has long been established that PPI therapy can alter intestinal microbial profiles (20). PPIs can directly affect intestinal microbiota through inhibition of the $\mathrm{H}^{+} / \mathrm{K}^{+}$ATPase which can inhibit microbial growth. Several bacteria, including H. pylori and S. pneumoniae, as well as fungi such as $C$. albicans, contain $\mathrm{H}^{+} / \mathrm{K}^{+}$ATPase in their plasma membranes which are highly homologous to their human counterparts (21). On the other hand, increase of intestinal $\mathrm{pH}$ can result in a diminished host defence against certain bacteria. Profound acid suppression increases the risk of enteric infections in susceptible individuals caused by Shigella, Salmonella, Yersinia or Clostridium difficile (22). The use of PPIs may also promote the expansion and colonization of $C$. difficile by its recognized potential to induce small bowel bacterial overgrowth with anaerobic colonic organisms (20). Many believe that PPI use may therefore directly contribute to $C$. difficile and other bacterial infections. Certainly, further research is needed to clarify the exact effects of PPIs on human intestinal microbiota. Nevertheless, alterations in intestinal microbiota should also be considered as a possible pathogenetic aspect in MC.

In conclusion, the observations of our case-control study confirm the presumed association between MC and PPI use and support the possible etiological role of PPI exposure in the development of MC. When patients are prescribed PPIs to treat upper gastrointestinal disorders, it is important to recognize that PPIs are interacting at multiple targets. As a result, MC may occur as an adverse reaction of PPIs and PPIs should be considered as a possible cause of diarrhea in this population. 
Appendix 1. Table 1.

\begin{tabular}{lccc} 
Drug & MC patients & Controls & OR [95\% CI] \\
\hline PPI & $37.9 \%$ & $12.6 \%$ & $4.5[2.0-9.5]$ \\
NSAID & $20.4 \%$ & $7.3 \%$ & $2.3[0.8-6.5]$ \\
BDZ & $12.8 \%$ & $8.4 \%$ & $1.2[0.4-3.7]$ \\
Diuretics & $12.8 \%$ & $8.4 \%$ & $1.4[0.5-4.4]$ \\
ACE-I & $7.7 \%$ & $5.3 \%$ & $1.8[0.5-4.4]$ \\
PPI+NSAID & $8.0 \%$ & $0 \%$ & N.A. \\
\hline
\end{tabular}

Table 1. Exposure to PPIs and NSAIDs in microscopic colitis (MC) patients and controls. 
Appendix 2. Table 2.

MC patients Controls p value $\left(\chi^{2}\right)$

\begin{tabular}{lccc}
\hline Celiac disease & $3.2 \%$ & $0 \%$ & 0.25 \\
Rheumatoid arthritis & $6.3 \%$ & $0 \%$ & 0.03 \\
Hypothyroidism & $5.2 \%$ & $0 \%$ & 0.06 \\
Irritable bowel syndrome & $8.4 \%$ & $6.3 \%$ & 0.78
\end{tabular}

Table 2. Comorbidities among microscopic colitis (MC) patients and controls. 
Appendix 3. Table 3.

6

7

\begin{tabular}{lcc} 
Type of PPI & MC patients & Controls \\
\hline Omeprazole & $40.0 \%$ & $50.0 \%$ \\
Esomeprazole & $22.8 \%$ & $30.0 \%$ \\
Pantoprazole & $28.6 \%$ & $20.0 \%$ \\
Rabeprazole & $5.7 \%$ & $0 \%$ \\
Lansoprazole & $2.8 \%$ & $0 \%$
\end{tabular}

Table 3. Types of PPIs used among microscopic (MC) patients and controls. 
References

1. Read NW, Krejs GJ, Read MG, Santa Ana CA, Morawski SG, Fordtran JS. Chronic diarrhea of unknown origin. Gastroenterology. 1980;78(2):264-71.

2. Pardi DS, Smyrk TC, Tremaine WJ, Sandborn WJ. Microscopic colitis: a review. Am J Gastroenterol. 2002;97(4):794-802.

3. Pardi DS, Loftus EV, Jr., Smyrk TC, et al. The epidemiology of microscopic colitis: a population based study in Olmsted County, Minnesota. Gut. 2007;56(4):504-8.

4. Tangri V, Chande N. Microscopic colitis: an update. J Clin Gastroenterol. 2009;43(4):293-6.

5. Fernandez-Banares $\mathbf{F}$, Esteve $\mathbf{M}$, Espinos JC, et al. Drug consumption and the risk of microscopic colitis. Am J Gastroenterol. 2007;102(2):324-30.

6. Chande N, Driman DK. Microscopic colitis associated with lansoprazole: report of two cases and a review of the literature. Scand J Gastroenterol. 2007;42(4):530-3.

7. Hilmer SN, Heap TR, Eckstein RP, Lauer CS, Shenfield GM. Microscopic colitis associated with exposure to lansoprazole. Med J Aust. 2006;184(4):185-6.

8. Thomson RD, Lestina LS, Bensen SP, Toor A, Maheshwari Y, Ratcliffe NR. Lansoprazoleassociated microscopic colitis: a case series. Am J Gastroenterol. 2002;97(11):2908-13.

9. Wilcox GM, Mattia AR. Microscopic colitis associated with omeprazole and esomeprazole exposure. J Clin Gastroenterol. 2009;43(6):551-3.

10. Beaugerie L, Pardi DS. Review article: drug-induced microscopic colitis - proposal for a scoring system and review of the literature. Aliment Pharmacol Ther. 2005;22(4):277-84.

11. Forgacs I, Loganayagam A. Overprescribing proton pump inhibitors. BMJ. 2008;336(7634):23.

12. van Boxel OS, Hagenaars MP, Smout AJ, Siersema PD. Socio-demographic factors influence chronic proton pump inhibitor use by a large population in the Netherlands. Aliment Pharmacol Ther. 2009;29(5):571-9.

13. Mullin JM, Gabello M, Murray $\mathbf{L}$, et al. Proton pump inhibitors: actions and reactions. Drug Discov Today. 2009;14(13-14):647-60.

14. Murray L, Gabello M, Rudolph DS, et al. Transmucosal gastric leak induced by proton pump inhibitors. Dig Dis Sci. 2009;54(7):1408-17.

15. Mullin JM, Valenzano MC, Whitby $\mathbf{M}$, et al. Esomeprazole induces upper gastrointestinal tract transmucosal permeability increase. Aliment Pharmacol Ther. 2008;28(11-12):1317-25.

16. Burgel N, Bojarski C, Mankertz J, Zeitz M, Fromm M, Schulzke JD. Mechanisms of diarrhea in collagenous colitis. Gastroenterology. 2002;123(2):433-43.

17. Tagkalidis PP, Gibson PR, Bhathal PS. Microscopic colitis demonstrates a T helper cell type 1 mucosal cytokine profile. J Clin Pathol. 2007;60(4):382-7.

18. Broeren MA, Geerdink EA, Vader HL, van den Wall Bake AW. Hypomagnesemia induced by several proton-pump inhibitors. Ann Intern Med. 2009;151(10):755-6.

19. Cundy T, Dissanayake A. Severe hypomagnesaemia in long-term users of proton-pump inhibitors. Clin Endocrinol (Oxf). 2008;69(2):338-41.

20. Lewis SJ, Franco S, Young G, O'Keefe SJ. Altered bowel function and duodenal bacterial overgrowth in patients treated with omeprazole. Aliment Pharmacol Ther. 1996;10(4):55761.

21. Vesper BJ, Jawdi A, Altman KW, Haines GK, 3rd, Tao L, Radosevich JA. The effect of proton pump inhibitors on the human microbiota. Curr Drug Metab. 2009;10(1):84-9.

22. Spiegel BM, Chey WD, Chang L. Bacterial overgrowth and irritable bowel syndrome: unifying hypothesis or a spurious consequence of proton pump inhibitors? Am J Gastroenterol. 2008;103(12):2972-6. 